Military Technical College

Kobry El-Kobba,

Cairo, Egypt

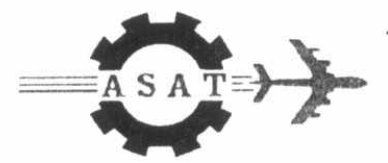

11-th International Conference

on Aerospace Sciences \&

Aviation Technology

\title{
SUPPRESSION OF VORTEX INDUCED VIBRATIONS ON CIRCULAR CYLINDER VIA BOUNDARY LAYER SUCTION
}

\author{
FAROUK M. OWIS*
}

\begin{abstract}
Marine risers used to convey oil from the sea bed to the sea level, marine cables, heat exchanger pipes, civil engineering structures and aircraft wings vibrate due to the formation of the vortex streets behind these structures. The interactions between the flow oscillations and the structure give rise to complicated vibrations of the structure which could cause structural damage due to the fatigue. Numerical simulation of the vortex-induced vibrations on circular cylinders is used to investigate the possibility of suppressing these vibrations for different engineering applications. The unsteady, incompressible, two-dimensional Navier-Stokes equations are solved numerically on a structured grid using the finite difference method. The effect of flow control using the boundary layer suction for fixed or moving cylinder is investigated by applying the appropriate boundary conditions on the cylinder surface. In the current study, natural motion of the cylinder is not considered. Flow oscillations are investigated only for fixed cylinder and for forced motion of the cylinder. The results indicate that the flow oscillations are completely damped for a fixed cylinder using suction on the cylinder surface. For forced motion of the cylinder, the vortex shedding from the surface is eliminated using the boundary layer suction. The flow oscillates only due to the forced motion of the body vibrations which means that the flow oscillations can be damped completely if the cylinder is left to oscillate naturally.
\end{abstract}

\section{KEYWORDS}

Fluid structure interaction, flow control, vortex shedding, upwind schemes, incompressible flow and numerical simulation

\footnotetext{
- Assistant Professor, Cairo University, Aerospace Engineering Department.
} 


\section{NOMENCLATURE}

C Artificial speed of sound

$D \quad$ Body diameter

$F \quad$ Frequency

$p \quad$ Pressure

Re Reynolds number

$\widetilde{R}_{T} \quad$ Turbulent Reynolds number

St Strauhal Number $S t=\frac{f D}{U}$

$t \quad$ Physical time

$U \quad$ Current velocity

$u_{1} \quad$ Velocity in $x_{1}$-direction

$u_{2} \quad$ Velocity in $x_{2}$-direction

$y \quad$ Normal distance from the wall to the grid point

$\beta \quad$ Artificial compressibility parameter

$\mu \quad$ Fluid viscosity

$\mu_{t} \quad$ Turbulent eddy viscosity

$v \quad$ Kinematic viscosity of the fluid

$v_{t} \quad$ Kinematic eddy viscosity

$\rho \quad$ Fluid density

$\tau \quad$ Artificial time

$\tau_{w} \quad$ Wall shear stress at the nearest grid point

\section{INTRODUCTION}

The fluid flow around circular cylinders or, more generally, bluff bodies leads to the formation of wake and to the vortex shedding from the body surface. As a result of the vortex formation and alternation from the top and bottom of the cylinder surface, periodic and asymmetric flow are generated which is well known as Von Karman vortexstreet. The alternate shedding of vortices in the near wake causes pressure and velocity fluctuations near the cylinder which in turn cause oscillating lift and drag forces on the body. The oscillating lift force is predominant, and if the body is free to move, it vibrates in a direction transverse to the ambient current. The amplitude of the cylinder oscillation can be of the order of one diameter and therefore present a potent source of fatigue as well as the possibility of clashing in multiple cylinder assemblies. In addition, the vortex induced vibration is of a significant engineering importance because it increases the drag coefficient which alters the static configuration, and thereby increases the static loading as reported by Every et al [1], Sarpkaya [2] and Yoerger et al [3]. The vortex induced vibrations on circular cylinders or bluff bodies can be found in many engineering applications such as marine risers used to convey oil from the sea bed to the sea level, marine cables, heat exchanger pipes, and civil engineering structures subjected to winds. Numerous experimental and numerical studies have been carried out on this fluid-structure interaction problem. Early studies concentrated on rigid structures in a cross flow [4-5]. Later investigations dealt with elastic structures where 
the structure natural frequency dominates the vortex shedding frequency, specially, if the two frequencies are very close to each other [6-9].

Analytical and numerical models have been proposed to evaluate the vortex-induced vibration problem. Analytical methods are used to model the periodic lift and drag forces on the cylinder such as those proposed by Hartlen [10], Skop [11], and Balasubraminain and Skop [12]. Numerical methods are also used to simulate this problem using the incompressible Navier-Stokes equations. Willden et al [8] predicted numerically the vortex induced vibration on long flexible circular cylinders using the vorticity-velocity formulation of the Navier-Stokes equations. The vorticity-streamfunction formulation is solved numerically by Zhou et al [13] to investigate the vortex-induced vibrations of an elastic circular cylinder. Other numerical efforts include solving the vorticity transport equation on a circular cylinder using a Lagrangian method [14-16]. This method is suitable for incompressible flows and it is naturally grid independent. The velocity in the flow field is calculated using the Biot-Savart law.

Many studies concentrated on investigating the nature of this phenomena and predicting the structure interaction to the vortex shedding problem. Few studies focused on controlling and suppressing the vortex induced vibrations. Different techniques have been suggested by some researchers to suppress the vortex induced vibrations. One of the methods used to suppress the flow-induced vibrations on circular cylinder is the periodic excitation of the flow. Hijima et al [17] investigated numerically the effect of the sound wave with specified frequency on the stability of the separated shear layer around the cylinder. In addition, experimental studies by Hijima et al [18] indicate that the vortex induced vibration of a circular cylinder can be suppressed using the stimulation of the separated shear layer by an acoustic wave with the frequency of the transition waves. Apparently, this method is examined only for small amplitude of the vortex-induced vibration. It is possible that the effect of periodic excitation on the larger amplitude vibrations might not be effective as explained by Hijima [17]. Moreover, the periodic excitation technique introduces high frequency oscillations at the cylinder surface which could be another source of fatigue stresses.

Optimal control of the vortex shedding from a fixed cylinder using suction and blowing on the body surface is investigated numerically by Zhijin et al [19] for a flow of very low Reynolds number $(\operatorname{Re}=110)$. The main purpose of their study is to determine the optimum locations and quantities of the suction and blowing by minimizing a cost function. In practice, the flow direction is not fixed since the ambient current changes its direction. Therefore, only uniform suction or blowing on the cylinder surface can suppress the vortex shedding regardless of the ambient current direction. In addition, Reynolds number associated with this flow is typically of order $10^{5}$. In the current study, suppression of the vortex shedding on circular cylinder is investigated numerically for high Reynolds number. The investigation includes suppression of the flow oscillations on fixed and moving cylinders using only suction. The effect of applying the boundary layer suction partially or totally on the cylinder surface is being compared to study the effective locations of the suction for fixed and forced motion cylinders. The fluid flow 
equations are solved numerically using the second-order upwind scheme of Roe [20] on a structured grid. The pressure-velocity formulation of the unsteady incompressible Navier-Stokes equations is used in the current study. In addition, one-equation turbulence model of Baldwin-Barth [21] is employed to compute the eddy vorticity of the flow field.

\section{GOVERNING EQUATIONS}

The unsteady, incompressible two-dimensional Navier-Stokes equations are used to simulate the vortex induced vibrations on circular cylinders. The governing equations are formulated using the pseudo compressibility method by adding an artificial time derivative of the pressure to the continuity equation. In addition, artificial terms are added to the momentum equations to simplify the numerical discretization using the upwind differencing scheme of Roe [20]. Therefore, the governing equations are written in the following form:

$$
\begin{aligned}
& \frac{1}{\beta} \frac{\partial p}{\partial \tau}+\frac{\partial u_{j}}{\partial x_{j}}=\theta \\
& \frac{\partial u_{i}}{\partial t}+\frac{\partial u_{i}}{\partial \tau}+\frac{\partial u_{i} u_{j}}{\partial x_{j}}=\frac{\partial p}{\partial x_{i}}+\frac{1}{\operatorname{Re}} \frac{\partial}{\partial x_{j}}\left[\left(\mu+\mu_{t}\right)\left(\frac{\partial u_{i}}{\partial x_{j}}+\frac{\partial u_{j}}{\partial x_{i}}\right)\right]
\end{aligned}
$$

The governing equations are normalized with respect to the fluid density, freestream axial velocity, and the cylinder diameter. Hence, the Reynolds number is defined as:

$\operatorname{Re}=\frac{U_{\infty} D \rho}{\mu}$

The governing equations can be rewritten in generalized curvilinear coordinates as follows:

$$
\begin{aligned}
& \Gamma_{e} \frac{\partial \hat{Q}}{\partial t}+\Gamma \frac{\partial \hat{Q}}{\partial \tau}+\frac{\partial\left(F-F_{v}\right)}{\partial \xi}+\frac{\partial\left(G-G_{v}\right)}{\partial \eta}=0 \\
& \hat{Q}=\frac{Q}{J}=\frac{I}{J}\left(P, u_{l}, u_{2}\right)^{T} \\
& F=\frac{1}{J}\left(U_{l}, u_{l} U_{l}+\xi_{x l} p, u_{2} U_{l}+\xi_{x 2} p\right)^{T} \\
& G=\frac{1}{J}\left(U_{2}, u_{l} U_{2}+\eta_{x l} p, u_{2} U_{2}+\eta_{x 2} p\right)^{T} \\
& U_{l}=\xi_{x l} u_{l}+\xi_{x 2} u_{2} \\
& U_{2}=\eta_{x l} u_{l}+\eta_{x 2} u_{2} \\
& F_{v}=\frac{\left(\mu+\mu_{t}\right)}{J \operatorname{Re}}\left(0,(\nabla \xi . \nabla \xi) \frac{\partial u_{1}}{\partial \xi}+(\nabla \xi . \nabla \eta) \frac{\partial u_{1}}{\partial \eta},(\nabla \xi . \nabla \xi) \frac{\partial u_{2}}{\partial \xi}+(\nabla \xi . \nabla \eta) \frac{\partial u_{2}}{\partial \eta}\right)^{T} \\
& G_{v}=\frac{\left(\mu+\mu_{t}\right)}{J \operatorname{Re}}\left(0,(\nabla \eta . \nabla \xi) \frac{\partial u_{l}}{\partial \xi}+(\nabla \eta . \nabla \eta) \frac{\partial u_{l}}{\partial \eta},(\nabla \eta \cdot \nabla \xi) \frac{\partial u_{2}}{\partial \xi}+(\nabla \eta \cdot \nabla \eta) \frac{\partial u_{2}}{\partial \eta}\right)^{T}
\end{aligned}
$$


The matrices $\Gamma_{e}$ and $\Gamma$ are given by

$$
\Gamma_{e}=\left[\begin{array}{lll}
0 & 0 & 0 \\
0 & 1 & 0 \\
0 & 0 & 1
\end{array}\right], \quad \Gamma=\left[\begin{array}{ccc}
\frac{1}{\beta} & 0 & 0 \\
0 & 1 & 0 \\
0 & 0 & 1
\end{array}\right]
$$

\section{TURBULENCE MODELING}

A one equation turbulence model of Baldwin and Barth [21], that avoids the need for an algebraic length scale, is employed to predict the turbulent flow over circular cylinders. This model is a simplified model of the standard $k-\varepsilon$ model. The model solves one transport equation for the turbulent Reynolds number $\widetilde{R}_{T}$ which is related to the eddy viscosity $v_{t}$. The transport equation is given by

$\frac{D\left(v \widetilde{R}_{T}\right)}{D t}=\left(c_{\varepsilon 2} f_{2}-c_{\varepsilon 1}\right) \sqrt{v \widetilde{R}_{T} P}+\left(v+\frac{v_{t}}{\sigma_{\varepsilon}}\right) \nabla^{2}\left(v \widetilde{R}_{T}\right)-\frac{1}{\sigma_{\varepsilon}}\left(\nabla v_{t}\right) . \nabla\left(v \widetilde{R}_{T}\right)$

where $D / D t$ is the substantial derivative and $P$ is the production term which is given by

$P=v_{t}\left(\frac{\partial u_{i}}{\partial x_{j}}+\frac{\partial u_{j}}{\partial x_{i}}\right) \frac{\partial u_{i}}{\partial x_{j}}$

The turbulent eddy viscosity is given by the following equation

$$
\begin{aligned}
v_{t} & =c_{\mu}\left(v \widetilde{R}_{T}\right) D_{l} D_{2} \\
D_{l} & =1-\exp \left(-y^{+} / A_{l}^{+}\right) \\
D_{2} & =1-\exp \left(-y^{+} / A_{2}^{+}\right) \\
y^{+} & =\frac{\sqrt{\tau_{w} / \rho}}{v} y
\end{aligned}
$$

The function $f_{2}$, which is used to introduce the transition from laminar to turbulent, is given by the following equation

$$
\begin{aligned}
f_{2} & =\frac{c_{\varepsilon l}}{c_{\varepsilon 2}}+\left(1-\frac{c_{\varepsilon l}}{c_{\varepsilon 2}}\right)\left(\frac{1}{\kappa y^{+}}+D_{1} D_{2}\right)\left(\sqrt{D_{1} D_{2}}+\frac{y^{+}}{\sqrt{D_{l} D_{2}}}\left(\frac{1}{A^{+}} \exp \left(-y^{+} / A^{+}\right) D_{2}\right.\right. \\
& \left.\left.+\frac{1}{A^{+}} \exp \left(-y^{+} / A_{2}^{+}\right) D_{l}\right)\right)
\end{aligned}
$$

The constants used in the model are given by

$\kappa=0.41, \quad c_{\varepsilon 1}=1.2, \quad c_{\varepsilon 2}=2.0, \quad c_{\mu}=0.09, \quad A^{+}=26, \quad A_{2}^{+}=10$

$\sigma_{\varepsilon}=\frac{\kappa^{2}}{\left(c_{\varepsilon 2}-c_{\varepsilon l}\right)} \frac{1}{\sqrt{c_{\mu}}}$ 


\section{NUMERICAL SCHEME}

\subsection{Convective Flux}

Upwind difference schemes are used to compute the convective flux derivatives. The flux difference splitting of Roe [20] scheme is used to discretize the convective terms. In order to use the upwind flux differencing schemes, the Jacobian matrices of the flux vectors are required in addition to their eigensystem. The flux vectors can be written in the following generalized vector form:

$F_{j}=\left[\begin{array}{l}U_{j} \\ u_{l} U_{j}+k_{x} p \\ u_{2} U_{j}+k_{y} p\end{array}\right]$

where $U_{j}=k_{x} u_{l}+k_{y} u_{2}, \quad k_{x}=\frac{1}{J} \frac{\partial \xi_{j}}{\partial x_{1}}, k_{y}=\frac{1}{J} \frac{\partial \xi_{j}}{\partial x_{2}}$ and $j=1,2$

The Jacobian matrix is given by

$\hat{A}=\frac{\partial F_{j}}{\partial Q}=\left[\begin{array}{lll}0 & k_{x} & k_{y} \\ k_{x} & \left(U_{j}+k_{x} u_{l}\right) & k_{y} u_{l} \\ k_{y} & k_{x} u_{2} & \left(U_{j}+k_{y} u_{2}\right)\end{array}\right]$

Using the preconditioning matrix $\Gamma$, we express the Jacobian matrix $\hat{A}$ as follows:

$A=\Gamma \Gamma^{-1} \dot{A}=\Gamma \tilde{A}$

$\widetilde{A}=\left[\begin{array}{lll}0 & \beta k_{x} & \beta k_{y} \\ k_{x} & U_{j}+k_{x} u_{l} & k_{y} u_{l} \\ k_{y} & k_{x} u_{2} & U_{j}+k_{y} u_{2}\end{array}\right]$

Thus, the eigenvalues of the preconditioning system $\widetilde{A}$ are $\lambda_{1}=U_{j}, \lambda_{2}=U_{j}+C, \lambda_{3}=U_{j}-C$

where $C=\sqrt{U_{j}^{2}+\beta\left(k_{x}^{2}+k_{y}^{2}\right)}$

The right eigenvector and the inverse of the right eigenvector matrices are given by

$$
\begin{aligned}
& X_{i}=\frac{1}{2 \beta C^{2}} \cdot\left[\begin{array}{ccc}
0 & \beta C & -\beta C \\
-2 \beta k_{y} & \left(u_{1} \lambda_{2}+\beta k_{x}\right) & \left(u_{1} \lambda_{3}+\beta k_{x}\right) \\
2 \beta k_{x} & \left(u_{2} \lambda_{2}+\beta k_{y}\right) & \left(u_{2} \lambda_{3}+\beta k_{y}\right)
\end{array}\right] \\
& X_{i}^{-1}=\left[\begin{array}{ccc}
k_{y} u_{l}-k_{x} u_{2} & -u_{2} \lambda_{l}-\beta k_{y} & -u_{1} \lambda_{l}+\beta k_{x} \\
-\lambda_{3} & \beta k_{x} & \beta k_{y} \\
-\lambda_{2} & \beta k_{x} & \beta k_{y}
\end{array}\right]
\end{aligned}
$$

The explicit part of the convective flux vectors is discretized using a third-order upwind difference scheme while the implicit part is approximated with a first-order upwind 
scheme. The flux difference splitting is constructed based on the eigenvalues and eigenvectors of the Jacobian matrix $\widetilde{A}$.

Considering the derivative of the convective flux in the $\xi$-direction, the following difference formula can be used:

$$
\begin{aligned}
& \frac{\partial F}{\partial \xi}=\frac{\widetilde{F}_{i+1 / 2}-\widetilde{F}_{i-l / 2}}{\Delta \xi} \\
& \widetilde{F}_{i+1 / 2}=\frac{1}{2}\left[F\left(Q_{i+1}\right)+F\left(Q_{i}\right)-\phi_{i+1 / 2}\right]
\end{aligned}
$$

where $\phi_{i+1 / 2}$ is the dissipation term.

For the first-order upwind scheme,

$$
\begin{aligned}
& \phi_{i+1 / 2}=\Delta F_{i+1 / 2}^{+}-\Delta F_{i+1 / 2}^{-} \\
& \Delta F_{i+1 / 2}^{ \pm}=\Gamma \widetilde{A}^{ \pm}(\bar{Q}) \Delta Q \\
& \bar{Q}=\frac{1}{2}\left(Q_{i+1}+Q_{i}\right) \\
& \Delta Q=Q_{i+1}-Q_{i}
\end{aligned}
$$

For the third-order upwind scheme,

$$
\phi_{i+1 / 2}=-\frac{1}{3}\left(\Delta F_{i-1 / 2}^{+}-\Delta F_{i+1 / 2}^{+}+\Delta F_{i+1 / 2}^{-}-\Delta F_{i+3 / 2}^{-}\right)
$$

\subsection{Time Discretization}

In the time accurate formulation, the physical time terms in the momentum equations are discretized using a second-order, three-point, backward difference formula as foilows,

$\Gamma_{e} \frac{\partial \hat{Q}}{\partial t}=\Gamma_{e} \frac{1.5 \hat{Q}^{n+l, k+1}-2 \hat{Q}^{n}+0.5 \hat{Q}^{n-1}}{\Delta t}$

where $n$ and $k$ are the indices of the physical and artificial time levels respectively.

The artificial time terms are replaced by the following implicit Euler finite-difference formula:

$\Gamma \frac{\partial \hat{Q}}{\partial \tau}=\Gamma \frac{\hat{Q}^{n+l, k+1}-\hat{Q}^{n+l . k}}{\Delta t}$

The governing equations (1-2) can be written in the following difference form:

$\left(\Gamma_{e} \frac{1.5}{J \Delta t}+\Gamma \frac{1}{J \Delta \tau}+\frac{\partial A_{j}}{\partial \xi_{j}}+\frac{\partial A_{j}^{v}}{\partial \xi_{j}}-S\right) \Delta Q=\Gamma_{e} \frac{1.5 \hat{Q}^{n+l, k+1}-2 \hat{Q}^{n}+0.5 \hat{Q}^{n-l}}{\Delta t}-\frac{\partial F_{j}}{\partial \xi_{j}}-\frac{\partial F_{j}^{v}}{\partial \xi_{j}}$

\subsection{Iterative Scheme}

Since, the left-hand of Eq. (36) is composed of an approximate Jacobian of the righthand side and the turbulence model is uncoupled from the mean flow equations, it is not useful to solve the resultant linear system exactly. Thus, it is enough to obtain an 
approximate solution to Eq. (36) in an efficient manner. Several methods could be used to solve this system iteratively including point relaxation, line relaxation and general matrix residual methods. In this work, the Gauss-Siedal line relaxation method is used to solve the resultant sparse matrix.

\section{BOUNDARY CONDITIONS}

Different boundary conditions are used in the simulations, including inflow, outflow and no-slip boundary conditions. All of the boundary conditions are treated implicitly in the code to reduce the restriction on the time step and to increase the stability of the code. For the inflow boundary, the velocities are specified, while the pressure is extrapolated from the interior points. At the outflow boundary, the pressure is specified, while the velocities are extrapolated. At the walls, the flow velocities are set equal to wall velocity plus the suction velocity, and the pressure is extrapolated. In addition, similar boundary conditions are used for the turbulent quantities.

\section{RESULTS}

The oscillating flow over a circular cylinder is investigated for a fixed and moving cylinder. The effect of using suction inside the boundary layer on the vortex formation and alternation for both the fixed and moving cylinders is studied. In all cases, a grid size of $180 \times 162$ in the axial and radial directions is used for the numerical descritization. A physical time step of 0.02 , artificial time step of 1 and artificial compressibility parameter of 4 are used in the simulations. A Reynolds number of $10^{5}$ is used in the simulations. Several computations are performed to investigate the possibility of suppressing the vibrations using suction on different parts of the cylinder surface. Table 1 presents the conditions of different suction cases on the cylinder surface.

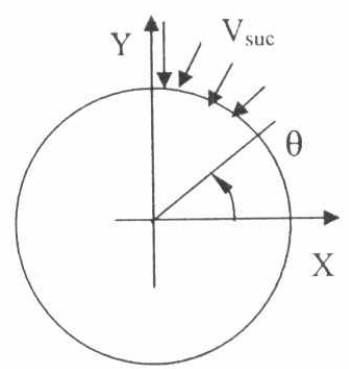

Fig. 1. Schematic layout of the cylinder showing the suction positions on the surface.

Fig. 2 presents the streamline contours and velocity vectors for the flow over a fixed cylinder without suction applied on the surface. The unsteady nature of the flow and the vortex formation behind the cylinder can be clearly seen in the graphs. As a result of this vortex formation the lift and drag forces oscillates on the cylinder surface even if the cylinder is being fixed. In Figs. 3-4, the time variation of the lift and drag coefficients on the cylinder surface is presented for cases presented in Table 1 . The results are compared with the case of no suction. In case of no suction, the drag coefficient 
Table 1. Simulation cases for the suction on the cylinder surface.

\begin{tabular}{|c|l|c|}
\hline $\begin{array}{l}\text { Case } \\
\text { No. }\end{array}$ & Surface subjected to suction & Suction velocity /Current velocity \\
\hline 1 & The whole cylinder surface & 0.04 \\
\hline 2 & The leeward direction of the cylinder $(x>0)$ & 0.04 \\
\hline 3 & The leeward direction of the cylinder $(x>0)$ & 0.025 \\
\hline 4 & $\begin{array}{l}\text { Portion of the upper and lower surface } \\
101^{0}<\theta<-60^{\circ} \text { and } 60^{\circ}<\theta<101^{0}\end{array}$ & 0.04 \\
\hline 5 & $\begin{array}{l}\text { Portion of the right half where } x>0.25 \text { or }- \\
60^{\circ}<\theta<60^{\circ}\end{array}$ & 0.04 \\
\hline
\end{tabular}

oscillates with a frequency which is double the frequency of the lift coefficient. This result is reported experimentally and numerically by many researchers. The amplitude of the lift coefficient oscillations is about 0.98 and the Strauhal number based on the cylinder diameter and the current velocity is 0.21 while the drag has a static component of 0.95 and unsteady component of 0.06 . Applying suction on the surface suppresses completely the flow oscillations as clear in cases 1 and 2 where the suction is applied whether on the whole surface or on the right half of the cylinder surface. In addition, the static component of the drag is significantly reduced to a minimal value. The flow oscillations are also reduced even after reducing the suction velocity from 0.04 to 0.025 .

a)

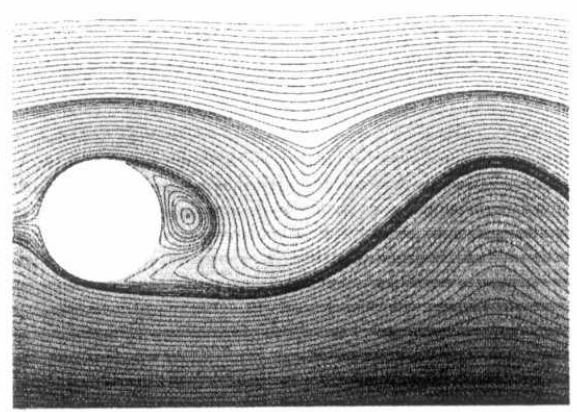

Fig. 2. Flow over a fixed cylinder no suction vectors. b)

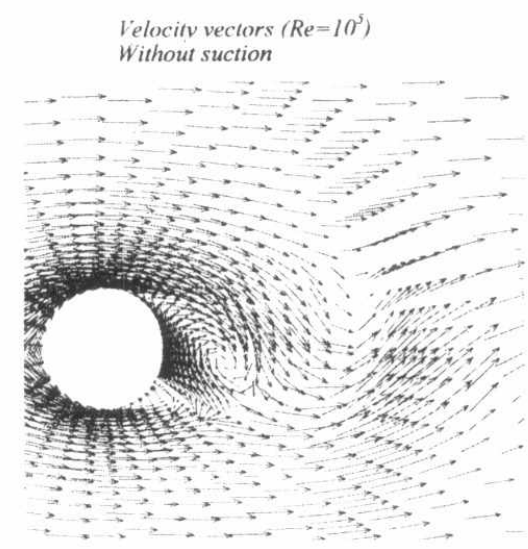

a) streamline contours

b) velocity

A small amount of suction applied all over the cylinder surface can be very effective in damping the flow oscillations for a fixed cylinder. This result is also clear from the velocity vectors presented in Fig. 5. Although suction is applied on the cylinder surface 
at particular positions as for cases 4 and 5, the flow oscillations exist and the suction has little effect on suppressing the vortex formation as clear from Fig. 4.
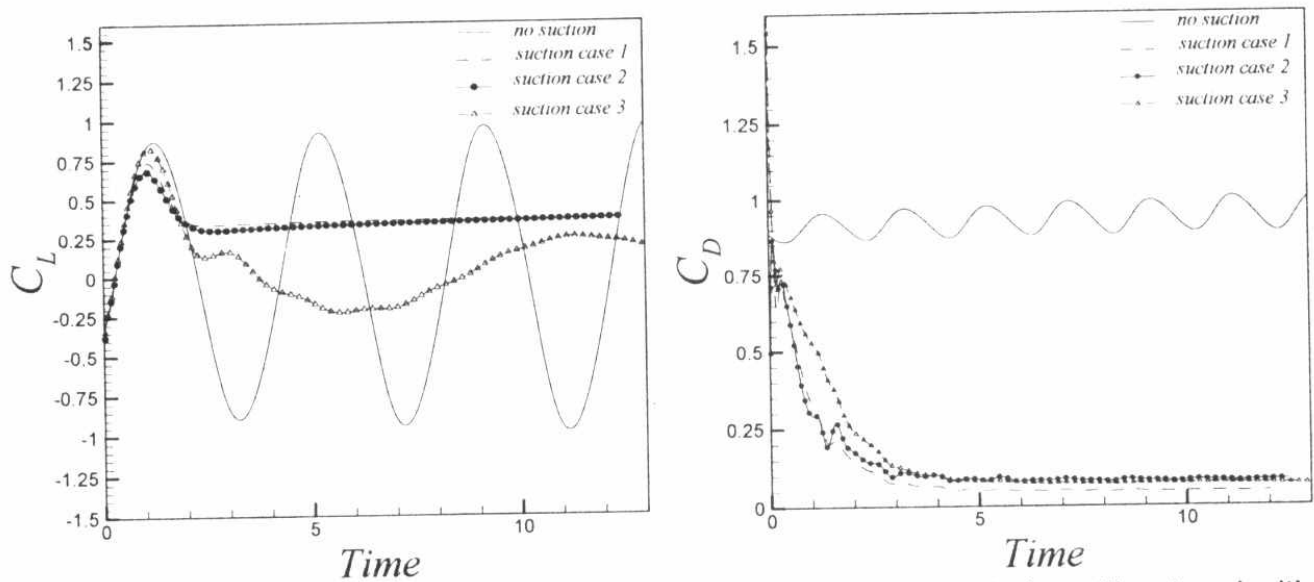

Fig. 3. Time variation of the lift and drag coefficients on a fixed cylinder without and with suction applied on different parts of the cylinder surface $\left(\operatorname{Re}=10^{5}\right)$.
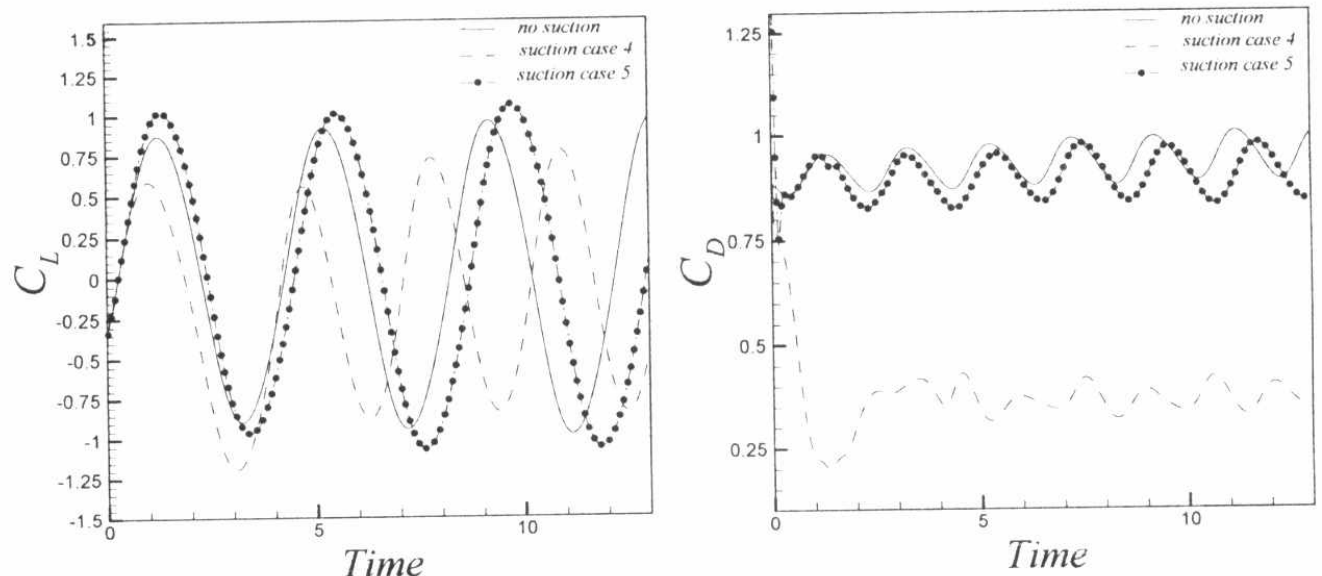

Fig. 4. Time variation of the lift and drag coefficients on a fixed cylinder without and with suction applied on different parts of the cylinder surface $\left(\operatorname{Re}=10^{5}\right)$. 

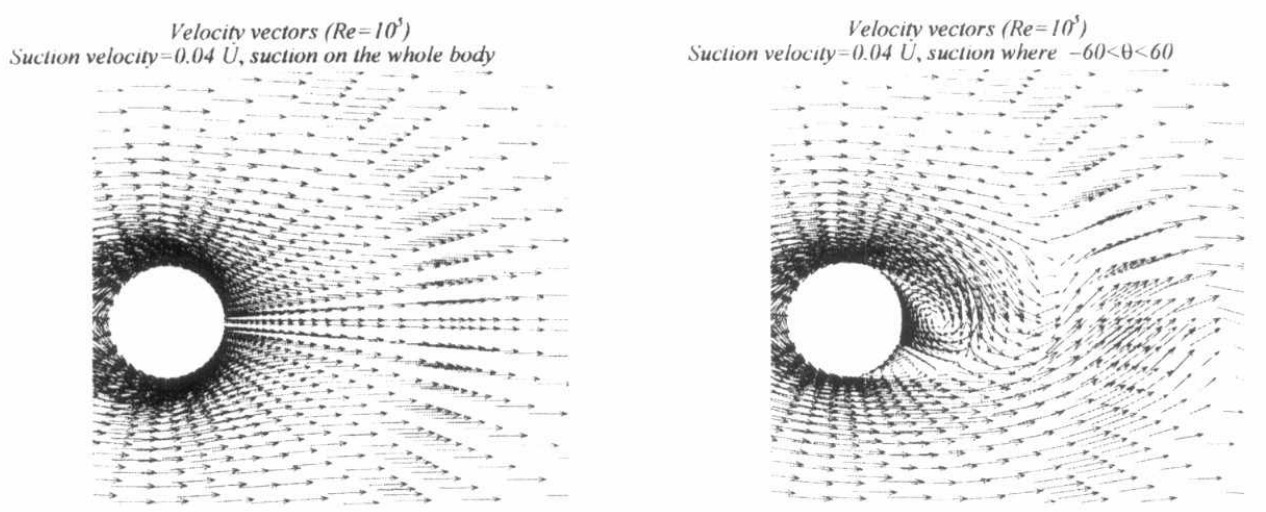

Fig. 5. Velocity vectors for the flow around fixed cylinder and suction applied at different positions on the surface $\left(\operatorname{Re}=10^{5}\right)$.

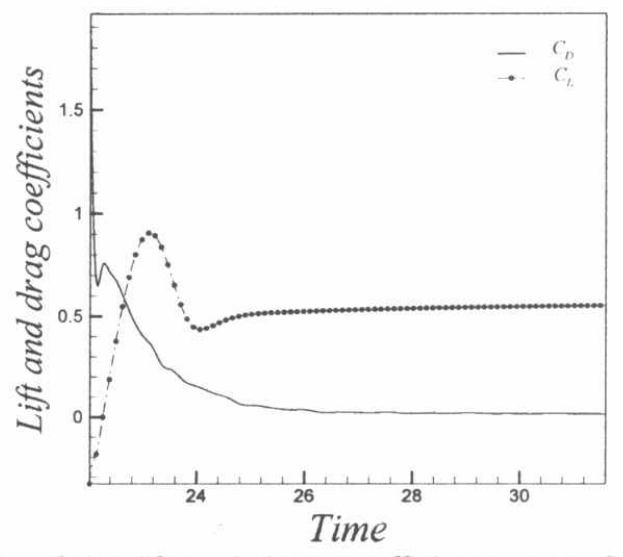

Fig. 6. Time variation of the lift and drag coefficients on a fixed cylinder with suction applied all over the cylinder surface $\left(R e=3 \times 10^{5}, V_{\text {suc }}=0.04 U_{\infty}\right)$.

The effect of the boundary layer suction is also investigated for Reynolds number higher than the critical Reynolds number $\left(\operatorname{Re}>2 \times 10^{5}\right)$ at which the wake behind the cylinder becomes turbulent. The results are presented in Fig. 6 for the lift and drag coefficient of the flow over a fixed cylinder at Reynolds number of $3 \times 10^{5}$. The results indicate that the flow oscillations are damped completely for suction speed of 0.04 the current velocity. In addition, the static drag on the cylinder is reduced to the value of the viscous drag. Therefore, the boundary layer suction can be very effective for controlling the vortex induced vibrations on structures even if the wake becomes turbulent. 
The effect of the cylinder motion on the flow oscillations with and without boundary layer suction is shown in Figs. $7-8$. The graphs indicate clearly to the interaction between the fluid and structure for this problem. Several frequencies are generated in the flow as a result of this interaction. In this particular case, the cylinder is forced to oscillate in the lift and drag directions with Strauhal numbers of 0.2 and 0.4 respectively. The oscillation velocities of the cylinder in the axial and radial direction respectively are given by the following equations:

$u_{l}=0.027 \sin (4 \pi * s t *$ time $) \quad, \quad u_{2}=0.13 \sin (2 \pi * s t *$ time $)$

where st is the Strauhal number which is assumed to be 0.2

Using the fast Fourier transform (FFT), the spectrum of the lift and drag coefficients are presented in Fig. 8. The figure shows several modes in the radial and axial directions are generated due to the cylinder motion in the absence of the boundary layer suction. The lift is dominated by two main components at Strauhal numbers of 0.2 and 0.23 which are the frequencies of the cylinder motion in the radial direction and of the vortex shedding. Several frequencies dominate the flow oscillations in the axial direction as clear from the spectrum of the drag coefficient. By examining the lift and drag coefficient after using the boundary suction, it is has been found that all the flow frequencies are eliminated except the frequency of the forced motion of the cylinder. So, only a frequency of 0.2 is present in the lift and a frequency of 0.4 is present in the drag which means the flow oscillations can be damped completely if the cylinder is left to oscillate naturally.
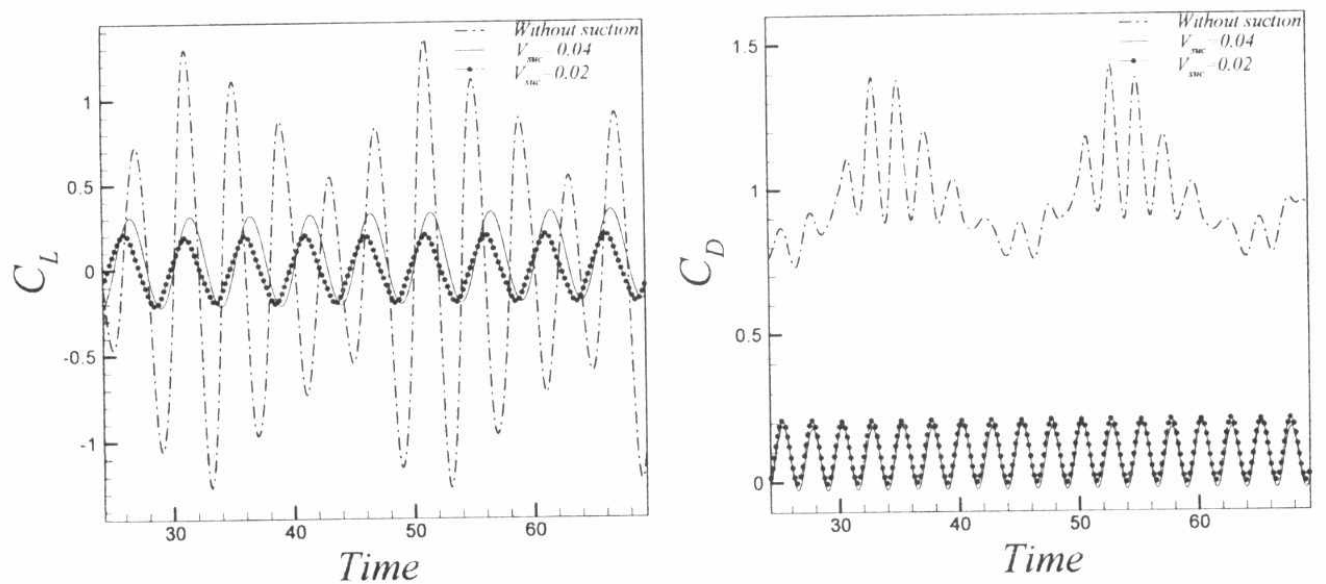

Fig. 7. Time variation of the lift and drag coefficients on a moving cylinder with different suction velocities applied all over the cylinder surface $\left(\operatorname{Re}=10^{5}\right)$. 

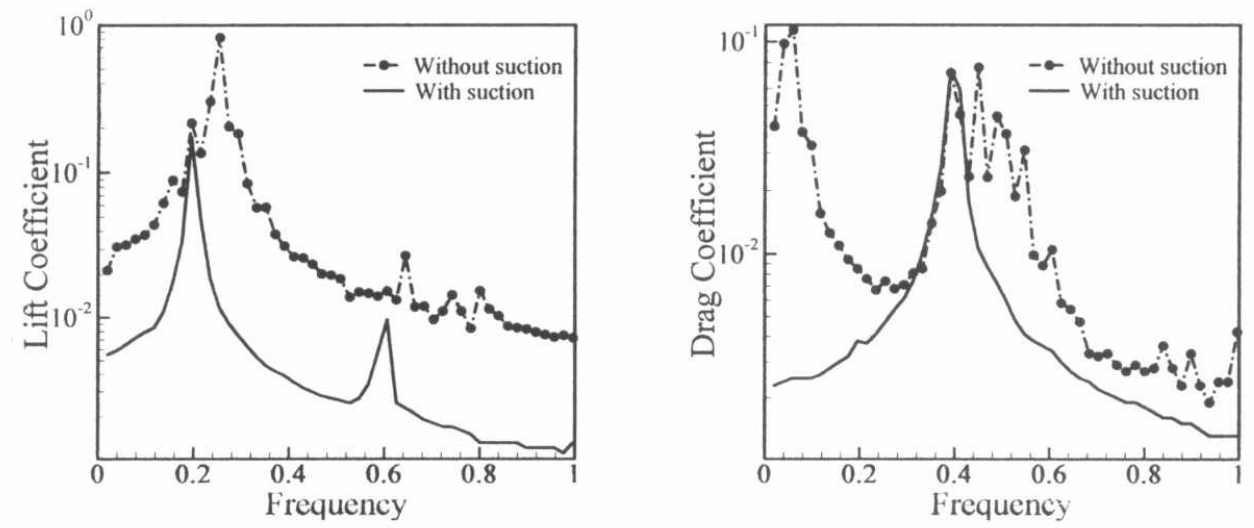

Fig. 8. Spectrum of the lift and drag coefficients for the moving cylinder with and without suction $\left(\operatorname{Re}=10^{5}, V_{\text {suc }}=0.02 U_{\text {. }}\right)$.

\section{CONCLUSIONS}

The control of the vortex induced vibrations on marine risers using the boundary layer suction is investigated numerically by solving the flow around fixed and moving cylinders. A finite difference code is developed to solve the unsteady two dimensional governing equations. The results indicate that a small amount of suction around the whole surface is very effective in damping the flow oscillations for fixed and moving structures and hence the structure interaction with the flow is reduced. The flow oscillates only due to the frequency of the forced motion of the cylinder. Therefore, the flow oscillations and the vortex shedding are attenuated using the boundary layer suction. Since, the natural oscillations of the cylinder are due to the flow oscillations and the vortex shedding, the natural motion of the cylinder can be completely damped if the flow oscillations are attenuated.

\section{REFERENCES}

1- Every, M.J, King, R. and Griffin, O.M, "Hydrodynamic loads on flexible marine structures due to vortex shedding", Proceedings of ASME Winter Annual Meeting, Washington, D.C., USA, (1981).

2- Sarpakaya, T., "Vortex-induced oscillations: a selective review", Journal of Applied Mechanics, Vol. 46, pp. 241-258, (1979).

3- Yoerger, D.R., Grosenbauch, M.A., Triantafyllou, M.S. and Burgess, J.J., "Drag forces and flow-induced vibrations of a long vertical tow cable-Part I: Steady state conditions", ASME Journal of Offshore Mechanics and Arctic Engineering, Vol. 113, pp. 30-36,(1991).

4- Richter, A. and Naudascher, E., "Fluctuating forces on a rigid circular cylinder in confined flow", Journal of Fluid Mechanics, Vol. 78, pp. 561-576, (1976). 
5- So, R.M.C. and Savkar, S.D., "Buffeting forces on rigid circular cylindersin cross flows", Journal of Fluid Mechanics, Vol. 105, pp. 397-425,(1981).

6- Feireisen, J.M., Montgomery, M.D. and Fleeter, S., "Unsteady aerodynamic forcing functions: a comparison between linear theory and experiment", Journal of Turbomachinery, Vol. 116, pp. 676-685, (1994).

7- West, G.S. and Apelt, C.J., "Fluctuating lift and drag forces on finite lengths of a circular cylinder in the subcritical Reynolds number range", Journal of Fluids and Structures, Vol. 11, pp. 135-158, (1997).

8- Willden, R.H.J. and Graham, J.M.R., "Numerical prediction of VIV on long flexible circular cylinders", Journal of Fluids and Structures, Vol. 15, pp. 659-669, (2001).

9- Kim, W.J. and Perkins, N.C., "Two-dimensional vortex-induced vibration of cable suspensions", Journal of Fluids and Structures, Vol. 16, No. 2, pp. 229-245, (2002).

10- Hartlen, R. and Currie, I., "Lift-oscillator model for vortex-induced vibrations", ACSE Journal of Engineering Mechanics, Vol. 96, pp. 577-591, (1970).

11-Skop, R.A., and Griffin, O.M.,"A model for the vortex-excited response of bluff cylinders", Journal of Sound and Vibration, Vol. 27, pp. 225-233, (1973).

12- Balasubramanian, S. and Skop, A.," A nonlinear oscillator model for vortex shedding from cylinders and cones in uniform and shear flows", Journal of Fluids and Structures, Vol. 10, pp. 197-214, (1996)

13- Zhou, C.Y., SO, R.M.C. and Lam K., "Vortex-induced vibrations of an elastic circular cylinder", Journal of Fluids and Structures, Vol. 13, pp. 165-189, (1999).

14- Chorin, A.J., "Numerical study of slightly viscous flow", Journal of Fluid Mechanics, Vol. 57, pp. 785, (1973)

15-Koumoutsakos, P. and Leonard A., "High-resolution simulations of the flow around an impulsively started cylinder using vortex methods", Journal of Fluid Mechanics, Vol. 296, pp. 1, (1995).

16-Leonard A., "Vortex methods for flow simulation", Journal of Computational Physics, Vol. 37, pp. 289, (1980).

17- Hiejjma, S., Nomura, T., Kimura, K. and Fujino, Y., "Numerical study on the suppression of the vortex-induced vibration of a circular cylinder by acoustic excitation", Journal of Wind Engineering, Vol. 67 and 68, pp. 325-335, (1997).

18- Hiejjma, S, Kimura, K. and Fujino, Y., "An experimental study on the effect of applied sound on the vortex-induced vibration of a circular cylinder", Proceedings of EASEC-5, pp. 1231-1236, (1995).

19-Zhijin, L., Navon, I.M., Hussaini, M.Y. and Dimet, F.X., "Optimal control of cylinder wakes via suction and blowing", Journal of Computers and Fluids, Vol. 32, pp. 149-171, (2003).

20-Roe, P.L., "Approximate Riemann solvers, parameter vectors, and difference schemes", Journal of Computational Physics, Vol. 43, pp. 357, (1981).

21-Baldwin, B. and Barth, T., "A one equation turbulence transport model for high Reynolds number wall-bounded flows", AIAA paper 91-0610, (1991). 